\title{
Discussion to the papers by G. N. Chandler and C. G. Clark
}

\author{
Chairman: Dr. J. B. Lynch
}

GILLESPIE. In the investigation of gastro-intestinal bleeding Dr Chandler mentioned that he uses the gastrocamera first and Professor Clark that he uses radiology first. Would you care to comment which is the best order to do these tests? Might you not obtain sufficient practical information from one test, say radiology alone, and might you not also discover some reason to make you withold passing the gastro-camera?

Chandler. We do both because we are interested in comparing their diagnostic efficacy. But I would agree with you that the barium meal is by far the least disturbing and if it doesn't give you sufficient information one should proceed to endoscopy. It is rare to meet any definite contraindication to endoscopy, the main one being a patient with a really rotten chest where one does not want to interfere with the cough reflex with a tube in the gullet.

HINTON. Do you get trouble in the acute barium meal with the artefacts due to blood in the stomach? Secondly, using the gastrocamera how soon can you get your results back?

Chandler. As to the first question you have to be a little careful here but blood clot is unlikely to simulate a chronic ulcer. As to the second question, the fibroscope camera is used routinely so that we can get immediate visualization. We don't wait for the films though we take them for record purposes.

HAMER. Have you found the camera of real value?

CHANDLER. Indeed yes, it has advanced so much on the old fibroscope it is like comparing a jet with a piston engine.

ClARK. I wonder if I could comment on this? While visualization is excellent with the fibroscope the quality of film obtained is not nearly so good.

Chandler. Vague purples seem to show.

Clark. This is true.

HADLEY. I have been using in the last 3 years the blind GT5 model and only recently the fibroscope camera and I agree the quality of films from the fibroscope camera is not as good as it ought to be but I haven't yet discovered why.

Chandler. How long does it take you to get a film back Dr Hadley?

HADLEY. Forty-eight hours. I cannot think of the reason for this as the same type of film is used. In haematemesis one cannot afford to wait $48 \mathrm{hr}$ for a result if you use the blind camera alone.

Swallow. Could I ask Dr Chandler to clarify two points, first, when considering hiatus hernia does one accept this as a cause of bleeding only if associated with acute ulcer or oesophagitis, as small hernias are often demonstrated in the ward barium meal?

CHANDLER. I agree the simple demonstration of a hernia is quite valueless; you must show either an intense oesophagitis or an ulcer by oesophagoscopy.

Swallow. I agree this is the usual view but the im- pression has got around that a hernia alone is an acceptable cause of bleeding. I didn't think it was.

CHANDLER. I couldn't agree more.

Swallow. When you have a good reason to believe that the patient has a chronic peptic ulcer on the basis of gastroscopic and radiological signs, you have said you favour early surgery. Recently I had a female patient who was only 17 but who had a deep chronic ulcer on the lesser curve of the stomach; would you push surgery in this type of case?

CHANDLer. I feel that bleeding chronic gastric ulcer is a surgical disease and surgery is nearly always required. Similarly in uncontrollable bleeding at any age one has to go straight ahead.

Swallow. In fact my young patient did settle down.

CHANDLER. In the patient who stops clearly one has to give some consideration to age. If you have a patient with a long history who is about the age of $\mathbf{5 0}$ but in whom bleeding has stopped it would be pointless to let them go out of hospital without an operation. Certainly in the gastric ulcer patient the indications are very great. In a girl of $17 \mathrm{I}$ would wait and see, realizing that the relapse rate in chronic gastric ulcer almost reaches $100 \%$ in 5 years or not quite.

SinGH. In a patient who had had a splenectomy and lienorenal shunt and bleeds again what treatment would you give?

CHANDler. I should send for Professor Sherlock! I have no experience of this type of case, little lienorenal work has been done in England, it was an operation devised by Linton and used mainly in Boston. It is a poor shunt compared with porto-caval, isn't it? In your case it might be possible to perform a porto-caval shunt.

SimPSON. If one has done an emergency partial gastrectomy for acute erosion and the patient continues to bleed, how do you manage this case?

Clark. As I have already said I would not do a partial gastrectomy and I don't think this business of 'blind gastrectomy' is advisable now. All I can say, in the last 2 years (and this may have been fortuitous as Dr Chandler has warned you against appraising small numbers of patients) we have not failed to find some adequate cause for the bleeding. If you do in fact do a 'blind gastrectomy' once the patient continues to bleed and the haemorrhage is such that you can't cope with it you have no option but to reoperate and either do a gastrotomy and try again to find the bleeding point. If again one failed one I suppose would have to do a total gastrectomy, which we know has been done by some surgeons, under these circumstances. I would be hesitant in relying purely on vagotomy.

Sircus. If I may come in on this hiatus hernia discussion, I was rather surprised to hear Dr Chandler's suggestion that the usual cause of bleeding was the oesophago-gastric hernia and not the para-oesophageal hernia. My experience is that bleeding requiring emergency resuscitation never is due to reflex oesophagitis and 
that in such circumstances it is coming possibly from a gastric ulcer in the herniated part of the stomach and certainly not from the oesophagus, whereas something like a third of the admissions for the complications of para-oesophageal hernia are because of massive intestinal bleeding; in my experience such bleeds are much more serious and difficult to manage.

CHANDler. This has not been our experience; we have a patient currently in the ward who was bleeding from a very intense oesophagitis and who required massive and continual transfusion and the gullet was seen to be full of blood.

ClaRK. I would agree with Dr Sircus here that when you see a patient with hiatus hernia who is bleeding sufficiently to require an operation you would look for another cause and the causes in our experience have not been oesophagitis but have been gastric ulcer and not infrequently erosions. This brings me onto something that $I$ might ask you: if you advocate conservative treatment for erosions, what do you do in the case of the massive bleeder when the barium meal shows hiatus hernia?

ChANDLER. I certainly would advise repair of the hernia, I would again like to emphasize that the hiatus hernia is not a common cause of major bleeding. I would persist with medical treatment unless the bleeding was so vicious as to require massive transfusion: then one has to think of surgery. A lot depends on the age of the patient. The younger ones do better with blood replacement and the older the patient the less well they do. You have to keep a close eye on the complications of the transfusion itself which adds to the difficulty here. The pressure is on you to do something, sometimes the wrong thing.

AtKinson. Can I ask Dr Sircus what the cause of bleeding was in the third of his patients with para-oesophageal hernia. It is not my experience that bleeding is a common complication in this type of hernia.

SIRCUs. You will appreciate that the cause of bleeding in most of those admitted with complications was a gastric ulcer, usually at the diaphragmatic level, but in some of them there was no sign of gastric ulcer, but profuse haemorrhagic gastritis associated with almost certainly a congestion from partial torsion.

Can I make one warning point with respect to this medical vagotomy? I have twice seen a myocardial infarction immediately following parenteral injection of Probanthine in these circumstances associated with the sudden onset of tachycardia, so that I think it may be a risky procedure.

MeIKLE. May I ask two questions? The first concerns the alarming problem of the middle-aged man with bronchitis and in whom an exacerbation in the winter seems to provoke haematemesis. Is it known whether the haematemesis is more likely to be fatal in bronchitics, and secondly have we still got only $48 \mathrm{hr}$ to try and-control the bleeding before surgery is entertained? Thirdly, how can we deal with what is not merely the reluctance of the surgeon, but the wretched man who turns his back and goes away?

The second question I would like to ask Dr Chandler is that I notice he still mentions morphine which I personally gave up a few years ago. I wonder if he or anyone else has any views on the use of chlorpromazine which I feel is a much more suitable drug and seems to suit haematemesis a great deal better than the opiates.

Clark. I'll answer part of the question as far as I can. The time limit of course is an individual one. But I think there is something very valuable one can do in the treatment of bleeding. Regardless of the state of the patient's lungs, and this is something I think described by Leroux, at the end of the operation or before if you like, push down a bronchoscope and you find little bits of clots. I think this is something that can be done to reduce the incidence of post-operative complications. As far as the chronic bronchitic is concerned, then you've obviously got to deal with him according to the severity of his bleeding and its cause; certainly if it is a mild bleed you feel that despite his previous history and all the rest of it that you should sway a little to conservatism here. Then if you are forced to operate I personally would wind up with a tracheostomy on such a patient to give me adequate control of his bronchial tree post-operatively.

Chandler. I would just add my agreement to this that although it is a formidable problem, tracheostomy, I think is the only way of getting some of these people through either before or after the operation. One may be enforced by the severity of the respiratory condition to do the tracheostomy first. We've had three such protlems: only two survived, and they surely wouldn't have done without tracheostomy and the bronchial toilet which this allows. With regard to your drug question, I agree about morphine, I think I said that I preferred phenobarbitone. I have no experience with chlorpromazine; I don't see why it shouldn't do as it's a good tranquillizer. I don't see that it should do any better than say barbiturate, and $I$ see no particular reason for using it in preference to barbiturate.

MeIKLE. Its main value is its antiemetic properties, patients cease to vomit. Could I repeat my first question? Do we know whether bronchitics who continue to bleed do very much worse than normal subjects?

Chandler. I think there is no doubt of this. Any associated disease adversely affects the mortality.

MeIKLE. Regardless of the method of treatment?

ChANDLER. Yes.

HANCOCK. This question of washing out the stomach: I think Capper suggests doing this under anaesthetic, with great resistance from the anaesthetists. Have you you had any trouble from the aspiration of blood into the bronchi following gastric lavage? Having done this washout have you done a gastroscopy and still found blood in the stomach?

CHANDLER. No we have had neither experience and have not gastroscoped the patient after lavage.

BEARN. Can you give us further information about patients with duodenal ulcer who bleed after conservative surgery? Can a case be made for doing conservative surgery on those with superficial ulcers and a gastrectomy on those ulcers which perforate into the pancreas?

Clark. I think this is probably the crux of the whole problem, the technical problem is considerable and we've had one or two hair-raising experiences. When I said we have dealt with all these patients by vagotomy and pyloroplasty this is not strictly true and in three patients the duodenal wall looked so 'tatty' at operation in fact I did a gastroenterostomy but there is no doubt this is some- 
thing which is different from dealing with the difficult duodenum when one is trying to do a gastrectomy. We find we really need a damn good exposure to do an adequate operation, but if we have that then we put great stitches with a heavy-bodied needle right through the actual ulcer; if you are the least bit timid about this, and don't in fact under-run the vessel, but simply try to close the ulcer bed, you will be in trouble. We had trouble when we only did this. But I think this can be adequate to control bleeding from nearly any ulcer and this has been the experience of one or two people from the States.

SAWYER. In relation to suture of the ulcer is there any justification in ligating the gastroduodenal artery?

Clark. Yes, I think there is. I think if I were dealing with a gastric ulcer, for example, I would invariably also ligate the left gastric artery, if one is dealing with an ulcer of the lesser curvature.

Atkinson. Could I ask Dr Chandler if he has any experience of arteriography, in identifying the site of haemorrhage which has been described in the American literature?

Chandler. No we haven't. We have considered using it in the diagnosis of obscure lesions in the stomach which Dr Herlinger will tell you about but have not used it in haematemesis because it is quite a formidable procedure in acutely ill people. To interrupt the routine work in the $\mathrm{X}$-ray department with an unannounced arteriogram is difficult.

I was interested in what Professor Clark said, that the effect of vagotomy is to divert blood from the mucosa. I can see that this would help in controlling the bleeding but I wondered if it wouldn't produce further ulcers in an anaemic vulnerable mucosa?

Clark. I have never seen an anaemic gastric mucosa! They say that erosions are associated with atrophic gastritis and an anaemic type of mucosa. I think the facts were well established by the Newcastle workers who showed vagotomy had this effect and certainly in view of the massive bleeds stopped by vagotomy something of this nature must occur. Perhaps of some interest in this respect is a recent suggestion by Butler from Bristol who in the case of erosions thinks that these are associated with reflux of the bile and he prefers in these patients to do a vagotomy rather than to anticipate the return of acid secretion, an antrectomy to reduce the most vulnerable area of bleeding and to finish up with a Roux-en-Y type of anastomosis to diminish biliary reflux. He makes a strong case for this and he is a man of considerable experience in the management of ulcer patients. It certainly seems worth exploring.

Another suggestion is that bleeding might be reduced by some fibrinolytic disorder so there might be some case for giving amincaproic acid (epsikapron) to some of these patients. Would you care to comment on that?

ChANDLER. I have no experience of this actually. Eighty per cent of patients with acute erosions are achlorhydric at the time of bleeding but in the majority of these acid secretion returns in a few days so that in many there is a history of antecendent emotional stress or disturbance far more commonly than is met in the chronic ulcer group. We haven't done this in any statistical way but we are working on it. I wondered if Barclay's work on gastric blood-flow just after the war showed that under conditions of emotional stress arteriovenous shunts opened up in the submucosa and diverted blood from the mucosa, indicated a situation in which aspirin could really produce marked erosive changes and bleeding. I have no experience of aminocaproic acid.

Question. Do you pass a large bore stomach tube and Senoran's evacuator on every patient who is bleeding?

Chandler. No I limit it to the elderly patient, the shocked patient when there is evidence from the acute barium meal of blood clot.

QUESTION. So the meal will have been done first then? Chandler. Yes.

HANCOCK. I doubt the ability of large tubes to evacuate the stomach, on several patients after vigorous gastric lavage with large bore tubes the stomach has been packed full of clot as large as a rugby football.

ChandLer. It would be most interesting to fibrescope these people afterwards and decide how effective lavage had been. 